\title{
EUROSCORE: still gold standard or less?
}

\author{
Christoph Bode $\cdot$ Malte Kelm
}

Received: 14 April 2009/Accepted: 17 April 2009/Published online: 5 May 2009

(C) Springer-Verlag 2009

Scoring systems have been developed in almost all subspecialties of clinical medicine. The major task of a scoring system is to systematically determine factors that may identify patients at risk, or determine efficacy of diagnostic and therapeutic procedures. They provide comparative measures between different sites and countries. Also, they facilitate the establishment of systems for quality assurance in a given treatment. Scoring systems are, however, never intended to substitute for clinical decision making of physicians in the context of an individual patient.

Ten years ago, in 1999, the "additive" EUROSCORE was published as a scoring system in cardiac surgery [6]. It was intended to assist in characterizing patients scheduled for cardiac surgery procedures. In an additive scoring system variables of comorbidities and cardiovascular function were semiquantitatively scored and then added up. This "simplified" additive scoring system was readjusted in 2003 by the introduction of a more precise logistic regression model, the so-called "logistic" EUROSCORE [9]. While the somewhat more detailed STS scoring system has been applied in North America the EUROSCORE became a popular benchmarking tool in Europe. In particular, it has proven to be very useful in assessing the management of patients by cardiac surgeons in European

\section{Bode}

Department of Internal Medicine III, Cardiology and Vascular Medicine, Universitätsklinikum Freiburg, Hugstetter Str. 55, 79106 Freiburg im Breisgau, Germany e-mail: Christoph.bode@uniklinik-freiburg.de

\section{Kelm (ه)}

Department of Internal Medicine B, Cardiology, Pulmonology and Vascular Medicine, Universitätsklinikum Düsseldorf, Moorenstr. 5, 40225 Düsseldorf, Germany e-mail: kelm@med.uni-duesseldorf.de; mkelm@ukaachen.de countries with substantial differences in their healthcare systems [8].

In the present paper, Gummert et al. [3] report on a subset of patients undergoing either isolated CABG or aortic valve replacement and tested the applicability of the additive and logistic EUROSCORE in this study population of an unaudited registry of the German society of Thoracic and Cardiovascular Surgery. The authors conclude "that the EUROSCORE insufficiently evaluates the risk of the current patient population and therefore should be carefully used as a tool for important therapeutic decision making". This raises several major questions: (1) what does risk assessment with the EUROSCORE mean? (2) what does "current patient population" reflect? (3) what does therapeutic decision making mean in this context?

Risk assessment with scoring systems in their analyses the authors underpin the importance of age in the risk assessment of patients undergoing medical procedures, which is well known and accepted in almost all medical disciplines. The present data are in line with the interpretation that mortality predicted by the EUROSCORE increases with increasing age, as is retrospectively analysis of mortality, further underscoring the value of the EUROSCORE. Nevertheless, many other factors such as left ventricular function, rhythm disorders and an array of pulmonary, vascular and metabolic comorbidities specifically affect the outcome of patients undergoing CABG or aortic valve replacement $[1,6]$. Unfortunately, the present paper does not provide mechanistic insights via a logistic regression model which parameter within the EUROSCORE exhibited the most valuable and which one the least useful information with respect to predictive quality. Also it might have been helpful to provide more detailed analysis on procedural data, such as off-pump versus on- 
pump surgery, time of extracirculatory support and so forth. What were the procedure related determinants of mortality? Readers should also be aware of the substantial differences in measures of in-hospital mortality, used in this registry, and 30-day mortality, used in the national BQS data base and most international randomized controlled trials in cardiovascular disease. This hampers exact comparisons on quality of procedures/outcome and, most importantly in this context, on the value and significance of the EUROSCORE system.

Patient population In the present study a cohort of 26.501 patients were analyzed, which represents approximately $25 \%$ of all patients that were treated in 2006 and 2007 in Germany. Seventeen (in 2006) and 29 (in 2007) centers, which are not identified, contributed to the data base. This raises several important questions: is there a selection bias in the centers contributing to the data base? Are there differences in high and low volume centers? In the present study, the reported figures on mortality differ from those reported in the cohort of patients in the current BQS report with $3.2 \%$ for isolated CABG procedures and $4.2 \%$ for isolated aortic valve replacement [7]. These differences add weight to the question, whether or not the patient population of the present study is representative. Most surprising in this context is the assessment of LV function, which is the most powerful predictor of mortality in almost all RCTs related to CVD [5, 10]: in the present study only $3.6 \%$ of the patients had a poor LV function. What made the difference between moderate and poor LV function and how were the respective measures defined and subsequently analyzed?

Therapeutic decision making The EUROSCORE was welcomed as a score in cardiac surgery to assess risk of patients undergoing coronary revascularization or valve replacement and has been accepted as a "gold standard" in Europe for many years [2, 4]. It was never intended to use the EUROSCORE as a substitute "tool" for therapeutic decision making of physicians in high risk patients as discussed by Gummert and coauthors. It now appears that with the advent of new treatment options, the EUROSCORE has become a liability. Rather than abolishing the EUROSCORE, it appears more adequate to update and develop the scoring system. It will certainly take further rigorously executed and representative registries to do just that. The authors are to be commended for having raised an important question and for having taken a first active step. Therapeutic decisions in elderly and high risk patients with a medical need for revascularization or valve replacement and reconstruction should always be made in close collaboration of cardiologists and cardiac surgeons considering not only cardiac but also all other comorbidities of the individual patient, as already is common practice in many centers in Germany. The upcoming new and complex therapeutic modalities of interventional valve replacement and reshaping should therefore be restricted only to those sites, where a close collaboration between both disciplines is ensured prior, during, and post interventional procedures.

\section{References}

1. Alexiou K, Kappert U, Staroske A, Joskowiak D, Wilbring M, Matschke K, Tugtekin SM (2008) Coronary surgery for acute coronary syndrome: which determinants of outcome remain? Clin Res Cardiol 97(9):601-608

2. Gogbashian A, Sedrakyan A, Treasure T (2004) EuroSCORE: a systematic review of international performance. Eur J Cardiothorac Surg 25(5):695-700

3. Gummert JF, Funkat A, Osswald B, Beckmann A, Schiller W, Krian A, Beyersdorf F, Haverich A, Cremer J (2009) EuroSCORE overestimates the risk of cardiac surgery: results from the national registry of the German Society of Thoracic and Cardiovascular Surgery. Clin Res Cardiol [Epub ahead of print]

4. Michel P, Roques F, Nashef SA, EuroSCORE Project Group (2003) Logistic or additive EuroSCORE for high-risk patients? Eur J Cardiothorac Surg 23(5):684-687

5. Nalysnyk L, Fahrbach K, Reynolds MW, Zhao SZ, Ross S (2003) Adverse events in coronary artery bypass graft (CABG) trials: a systematic review and analysis. Heart 89(7):767-772

6. Nashef SA, Roques F, Michel P, Gauducheau E, Lemeshow S, Salamon R (1999) European system for cardiac operative risk evaluation. Eur J Cardiothorac Surg 16(1):9-13

7. Qualitätsreport Leistungsbereich Herzchirurgie (2007) BQS Bundesgeschäftsstelle Qualitätssicherung $\mathrm{gGmbH}$. http://www. bqs-qualitaetsreport.de/2007/ugebmisse/down/qr2007.pdf

8. Roques F, Nashef SA, Michel P, Pinna Pintor P, David M, Baudet E (2000) Does EuroSCORE work in individual European countries? Eur J Cardiothorac Surg 18(1):27-30

9. Roques F, Michel P, Goldstone AR, Nashef SA (2003) The logistic EuroSCORE. Eur Heart J 24(9):881-882

10. Tjang YS, van Hees Y, Körfer R, Grobbee DE, van der Heijden GJ (2007) Predictors of mortality after aortic valve replacement. Eur J Cardiothorac Surg 32(3):469-474 